\title{
Mortality Outcomes of Endovascular Treatment and Surgical Clipping in Patients with Cerebral Aneurysms: A Single-Center Study
}

\author{
Abdullah SUKUN 1 , Mustafa CETIN², Ahmet ALPARSLAN², Ramazan UYAR ${ }^{3}$, Tolga GEDIZ³ ${ }^{3}$ Bulent CEKIC², \\ Mert KOROGLU² \\ ${ }^{1}$ Kars Harakani State Hospital, Department of Radiology, Kars, Turkey \\ ${ }^{2}$ Antalya Education and Research Hospital, Department of Radiology, Antalya Turkey \\ ${ }^{3}$ Antalya Education and Research Hospital, Department of Neurosurgery, Antalya Turkey \\ This study has been presented at the $11^{\text {th }}$ Annual Interventional Radiology Meeting, the first results were presented as an oral presentation. Results were \\ accepted as a eposter at the $39^{\text {th }}$ National Radiology Congress.
}

Corresponding author: Abdullah SUKUN abdullah.sukun@gmail.com

\section{ABSTRACT}

AIM: To compare endovascular and surgical treatment methods for cerebral aneurysms focusing on mortality.

MATERIAL and METHODS: The study included 187 patients who had undergone aneurysm treatment. The patients were divided into four groups according to their treatment modality and subarachnoid hemorrhage status: patients with endovascular treatment and bleeding aneurysms (EVG-b), patients with endovascular treatment and non-bleeding aneurysms (EVG-nb), patients with surgical clipping and bleeding aneurysms (SCG-b), and patients with surgical clipping and non-bleeding aneurysms (SCG-nb). The Hunt-Hess scores, Fisher grade, aneurysm morphology, and length of stay (LOS) were compared between groups.

RESULTS: There was no significant difference in the mortality rate between EVG-b and SCG-b at the end of the first year (23.5\% and $39.7 \%$, respectively; $p>0.05$ ). A significantly shorter LOS was observed in EVG-b than in SCG-b (11.5 days and 15 days, respectively; $\mathrm{p}=0.027$ ). Fusiform aneurysms were associated with higher patient mortality, whereas saccular aneurysms were associated with a 1.9-fold higher survival ( $p=0.037 ; 95 \%$ confidence interval: $0.83-4.74)$. The rate of closure of non-bleeding aneurysms was $93.4 \%$. Complete embolization was verified in all bleeding aneurysms. In EVG-nb, the morbidity rate was $5 \%$, the mortality rate was $3 \%$, and the mean LOS was 2.86 days.

CONCLUSION: Both treatment methods showed similar mortality rates, but hospital stays were shorter after endovascular treatment.

KEYWORDS: Cerebral aneurysm, Surgical clipping, Endovascular treatment, SAH

ABBREVIATIONS: SAH: Subarachnoid hemorrhage, LOS: Length of hospital stay, EVG: Endovascular group, SCG: Surgical clipping group

Abdullah SUKUN (1): 0000-0003-0047-0857

Mustafa CETIN (1) : 0000-0002-3506-2039

Ahmet ALPARSLAN (1) : 0000-0001-7083-5618
Ramazan UYAR (10) : 0000-0003-2304-4586

Tolga GEDIZ (1) : 0000-0001-6277-9847

Bulent CEKIC (D) : 0000-0001-5031-5260
Mert KOROGLU (1) : 0000-0003-1937-5761 


\section{- INTRODUCTION}

S ubarachnoid hemorrhage (SAH) is a mortal complication due to cerebral aneurysms. Aneurysms are typically treated with two methods: endovascular coil embolization and surgical clipping. Both treatment methods aim to prevent re-bleeding by providing permanent occlusion. It remains controversial which treatment method is superior. The International Subarachnoid Aneurysm Trial (ISAT), a multicenter randomized controlled trial published in 2002 (18), showed that mortality rates at the end of the first year were significantly lower in the endovascular treatment group. After the ISAT study, a paradigm towards the endovascular treatment method emerged. The advantages and disadvantages of both treatments were reported in the 5-10 years of follow-up. However, mortality rates were similar (1).

In 2012, the Barrow Ruptured Aneurysm Trial (BRAT) found lower mortality rates in the endovascular treatment group in the early period, at the end of the first year, than in the surgical clipping group (16). Research indicates that both treatment methods are complementary, and the treatment approach should be decided according to the patient. A meta-analysis published in 2020 (11) reported that surgical clipping had a higher incidence of complications than endovascular treatment in non-bleeding aneurysms, but clipping was associated with a lower risk of death than endovascular treatment in bleeding aneurysms. However, no significant difference was found in patient mortality non-bleeding aneurysms. The risk of hydrocephalus and residue was lower in clipping treatment than in endovascular treatment. The risk of ischemic infarction and vasospasm did not differ significantly between the two treatment methods. Another study (17) reported that patients treated with endovascular treatment had a higher re-treatment rate than those treated with surgical clipping. Bleeding and rerupture rates were similar in both groups.

Using the data in our center, we aimed to test the hypothesis that both treatment methods are safe and there is no significant difference in mortality.

\section{- MATERIAL and METHODS}

All procedures performed in the studies involving human participants were in accordance with the ethical standards of the institutional and/or national research committee and with the 1964 Helsinki Declaration and its later amendments or comparable ethical standards. Institutional review board approval for this study was obtained in April 2016. Informed consent was obtained from all individual participants included in the study.

Endovascular aneurysm treatment started at our institution in 2014. All aneurysm treatments performed between 2012 and 2016 were included in the study. A total of 20 patients who did not accept treatment despite the detection of aneurysms, who were referred to another center, or who could not undergo the endovascular treatment procedure were excluded from the study. In addition, six patients who underwent endovascular aneurysm treatment and seven patients who underwent surgical clipping were excluded from the study because they could not be followed up at the end of the first year.

In total, 187 patients who were treated for aneurysms were included in the study. The patients were divided into two groups according to the type of treatment: endovascular treatment group (EVG) and surgical clipping group (SCG). Patients were also divided into two subgroups according to $\mathrm{SAH}$ status (bleeding [b] and non-bleeding [nb]). However, surgical clipping was performed on patients with SAH. There were no patients who underwent elective surgical clipping. The study evaluated 130 aneurysms in 109 patients who were treated with endovascular treatment between 2014 and 2016 and 100 aneurysms in 78 patients who were treated with surgical clipping between 2012 and 2016. In total, 69 male and 118 female patients were included, aged between 19 and 89 years. Patient characteristics and demographic data are presented in Table I and II.

In patients who underwent surgical clipping between 2012 and 2014, aneurysms were diagnosed by computed tomography angiography (CTA). After the angiography unit was established, an interventional radiology clinic was opened, and all aneurysms were diagnosed by digital subtraction angiography (DSA). The decision about the treatment process was taken in separate sessions by neurosurgeons and interventional radiologists as a Council decision. Signed consent forms were obtained from all patients undergoing endovascular aneurysm treatment. All procedures were performed with a Philips Allura FD20 angiography device (@) Koninklijke Philips N.V., The Netherlands) available in our unit. Aneurysm treatments were performed under general anesthesia using modern anesthesia techniques. The aneurysm treatment started with

Table I: Patients Data

\begin{tabular}{cccccc}
\hline \multicolumn{2}{c}{ Hunt Hess Grade } & \multicolumn{2}{c}{ Fisher Grade } & \multicolumn{2}{c}{ Length of hospital stay } \\
\hline $\mathrm{HH} 1$ & $9(8 \%)$ & $\mathrm{F} 1$ & $11(9.8 \%)$ & $<2$ days & $29(15.5 \%)$ \\
\hline $\mathrm{HH} 2$ & $39(34.8 \%)$ & $\mathrm{F} 2$ & $19(16.9 \%)$ & 3 days & $20(10.7 \%)$ \\
\hline $\mathrm{HH} 3$ & $35(31.2 \%)$ & $\mathrm{F} 3$ & $41(36.6 \%)$ & 4 days & $17(9.1 \%)$ \\
\hline $\mathrm{HH} 4$ & $21(18.7 \%)$ & $\mathrm{F} 4$ & $41(36.6 \%)$ & $5-9$ days & $29(15.5 \%)$ \\
\hline $\mathrm{HH} 5$ & $8(7.1 \%)$ & & & $10-19$ days & $55(29.5 \%)$ \\
\hline & & & & $>20$ days & $37(19.8 \%)$ \\
\hline
\end{tabular}


the Seldinger method, from one or two groins, by inserting a $6 \mathrm{~F}$, long neurovascular sheath. The internal carotid artery was selectively catheterized, using appropriate guidewires, a vertebral catheter, and a distal access catheter. Suitable microguidewires and microcatheters were used to catheterize the aneurysm sacs. To occlude the aneurysms, stents, balloons, flow diverters, intra-aneurysmal flow-modulating devices, and microcoils were used. After treatment, neurological examination was performed. After the procedure, a vascular closure device was used to close the artery, and the patients were transferred to intensive care. In SCG, the aneurysm neck was reached with an appropriate approach for each patient, and clipping was performed. Postoperative control computed tomography was performed for each patient 2-3 $\mathrm{h}$ after surgical clipping.

All patients were followed up 1, 6 and 12 months after treatment. Follow-up procedures in the endovascular group included magnetic resonance angiography (MRA) at 1 month, CTA at 6 months, and MRA at 12 months post treatment. In SCG, follow-up was performed using DSA at 1 month, CTA at 6 months, and MRA at 12 months post treatment. In both groups, patients were evaluated in terms of SAH; aneurysm location, size, and morphology; Hunt-Hess score and Fisher grade; length of stay (LOS); and post-treatment mortality on the basis of the hospital archives.

\section{Statistical Analysis}

All statistical analysis was performed with SPSS 25.0 (Statistical Package for Social Sciences for Windows, Chicago, IL). Categorical variables are presented as numbers and percentages, whereas continuous variables are presented as mean \pm standard deviation or median. For comparisons of categorical variables, Pearson's chi square test, Yates corrected chi square test, and Fisher's exact test were used. Continuous variables were analyzed according to the normality assessment using Kolmogorov-Smirnov and Shapiro-Wilk tests. The Mann-Whitney U test was applied to data with nonnormal distribution to perform binary comparisons between independent groups. Significant differences between groups were assessed with the Kruskal-Wallis test as the data were not normally distributed. In all analyses, the significance level was set to $p<0.05$.

\section{RESULTS}

The average age of the patients was $54.65 \pm 13.90$ (min 19max 89$) ; 63 \%$ of the patients were women $(n=118)$ and $37 \%$ were men $(n=69)$. Subarachnoid hemorrhage was detected in 34 patients in EVG-b and 78 patients in SCG-b-a total of 112 patients. In total, 94 non-bleeding aneurysms in 75 patients were treated endovascularly (EVG-nb). No patients with non-bleeding aneurysms underwent surgical clipping (SCG-nb; $n=0$ ). There was no significant difference in mortality rates between EVG-b and SCG-b at the end of the first year (23.5\% and $39.7 \%$, respectively; $p>0.05$, Table III). High Hunt-Hess score and Fisher grade were associated with mortality. Regarding morphology, fusiform aneurysms were associated with higher patient mortality. Saccular aneurysms were associated with a 1.9 -fold higher survival $[p=0.037$; 95\% confidence interval $(95 \% \mathrm{Cl}): 0.83-4.74]$. Irregularity in the aneurysm contours, referred to as a nipple, is a sign of impending rupture. No significant correlation was found between the detection of an aneurysm nipple and mortality (Table IV).

Ninety-two aneurysms were examined in EVG-nb at the end of the first year. Eighty-six of these aneurysms were completely closed, whereas residual aneurysms were observed in six of them. The rate of closure of non-bleeding aneurysms was 93.4\%. Complete embolization was verified in all bleeding aneurysms.

The average LOS was 2.86 days in EVG-nb. A significantly lower hospital stay was observed in EVG-b than in SCG-b ( 11.5 days and 15 days, respectively; $p=0.027$ ). There was no statistically significant difference in the LOS between patients

Table II: Aneurysms Data

\begin{tabular}{lcc}
\hline Aneurysm location & & \\
\hline AcomA & 73 & $31.8 \%$ \\
\hline PcomA & 23 & $10 \%$ \\
\hline ICA & 50 & $21.8 \%$ \\
\hline MCA & 71 & $30.9 \%$ \\
\hline BA & 9 & $3.9 \%$ \\
\hline VA & 2 & $0.8 \%$ \\
\hline Others & 2 & $0.8 \%$ \\
\hline Total & 230 & \\
\hline
\end{tabular}

Aneurysm size $(\mathrm{mm})$

\begin{tabular}{lcc}
\hline Baby Aneurysm <3 mm & 1 & $0.4 \%$ \\
\hline Small Aneurysm 3-6 mm & 115 & $50 \%$ \\
\hline Medium Aneurysm 7-10 mm & 74 & $32.2 \%$ \\
\hline Big Aneurysm 11-25 mm & 36 & $15.7 \%$ \\
\hline Giant Aneurysm >25 mm & 4 & $1.7 \%$ \\
\hline Mean & $7.7 \pm 4.94$ & \\
\hline Min-Max & $2-30 \mathrm{~mm}$ & \\
\hline
\end{tabular}

Treatment methods

\begin{tabular}{lrc}
\hline Surgical Clipping & 100 & $43.5 \%$ \\
\hline Coiling & 28 & $12.2 \%$ \\
\hline Balloon- Assisted Coiling & 14 & $6.1 \%$ \\
\hline Stent-Assisted Coiling & 28 & $12.2 \%$ \\
\hline Flow Diverter & 42 & $18.3 \%$ \\
\hline Flow Diverter and Coiling & 9 & $3.9 \%$ \\
\hline Flow Disruption & 9 & $3.9 \%$ \\
\hline
\end{tabular}


Sukun A. et al: Mortality Outcomes of EVT and Surgical Clipping

Table III: Comparison of EVG and SCG in Terms of Mortality and LOS

\begin{tabular}{|c|c|c|c|}
\hline & EVG & SCG & $\mathbf{p}$ \\
\hline Nonbleeding & 75 & 0 & \\
\hline Patients & EVG-b & SCG-b & \\
\hline Bleeding & 34 & 78 & \\
\hline Living patients & $26(76.5 \%)$ & $47(60.3 \%)$ & \\
\hline Length of Hospital Stay & 11.50 day & 15.00 day & 0.027 \\
\hline
\end{tabular}

Table IV: Factors Affecting Mortality in SAH Patients

\begin{tabular}{|c|c|c|c|}
\hline & Live Patients & Dead Patients & $\mathbf{p}$ \\
\hline I (9) & $6(66.7 \%)$ & $3(33.3 \%)$ & \\
\hline II (39) & 30 (76.9\%) & $9(23.1 \%)$ & \\
\hline III (35) & $26(74.3 \%)$ & $9(25.7 \%)$ & \\
\hline $\mathrm{V}(8)$ & $2(25.0 \%)$ & $6(75.0 \%)$ & \\
\hline Fisher Grade & & & 0.030 \\
\hline $1(11)$ & $11(100 \%)$ & $0(\% 0)$ & \\
\hline $2(19)$ & $12(63.2 \%)$ & $7(36.8 \%)$ & \\
\hline \multicolumn{4}{|c|}{ Aneurysm Morphology } \\
\hline Saccular (27) & $23(85.2 \%)$ & $4(14.8 \%)$ & 0.037 \\
\hline Fusiform (7) & $3(47.9 \%)$ & $4(57.1 \%)$ & \\
\hline Nipple+ & $10(90.9 \%)$ & $1(9.1 \%)$ & 0.220 \\
\hline Nipple- & $16(69.6 \%)$ & $7(30.4 \%)$ & \\
\hline
\end{tabular}

with fusiform and saccular aneurysms or patients with and without irregularities in the aneurysm margins (saccular, 15.19 days; fusiform, 12 days; Nipple+, 15.09 days; Nipple-, 14.26 days; $p>0.05$ ).

\section{DISCUSSION}

This study revealed that the most crucial factor affecting mortality in aneurysm treatment is the occurrence of SAH. Hunt-Hess scores and Fisher grade affected patient mortality among patients with $\mathrm{SAH}$; however, the patient mortality rate did not differ according to whether the aneurysms were treated using the endovascular or surgical method. Previous randomized studies have shown that the mortality rates of patients with $\mathrm{SAH}$ at the end of the first year did not differ between treatment groups (7). Long-term follow-up of patients treated for aneurysms who experienced SAH showed that the rates of death and sequela were higher in patients who underwent surgical clipping. In previous studies, the recurrent bleeding rate during the late period was found to be higher among patients in EVG (19). In our study, mortality rates were evaluated at the end of the first year, and no significant difference was found between the two treatment groups.

Another study reported similar mortality rates in the third year of treatment in both groups. However, aneurysm closure rates were lower in EVG than in SCG; EVG patients often required repeated surgical treatment. In the present study, the residual risk of anterior circulation aneurysms was lower in SCG than in EVG. Endovascular treatment has been reported to be 
advantageous in terms of mortality and morbidity in posterior circulation aneurysms (22). In our study, no residual risk was detected in patients with $\mathrm{SAH}$.

Previously, the prevalence of cerebral aneurysms was reported to be $3 \%$, and an increased risk of aneurysm and $\mathrm{SAH}$ was reported for women older than 50 years (23). In our study, the average age was 54.6 years. Sixty-three percent of the patients with aneurysm were women, and SAH was more common in women. In the International Study of Unruptured Intracranial Aneurysms (ISUIA), the annual morbidity rate was reported to be $6.4 \%$, and the mortality rate was $3.1 \%$ (3). In our study, the mortality and morbidity rates in EVG-nb were 3\% and $5 \%$, respectively. In our hospital, endovascular treatment is preferred as the primary treatment option for patients with non-bleeding aneurysms. Therefore, we lack data regarding the mortality and morbidity rates associated with the surgical treatment of non-bleeding aneurysms.

McDonald et al. compared 3,551 coil embolization and 1,388 surgical clipping treatments. Mortality rates were found to be similar in both groups, which is consistent with the findings in our study. A previous study reported a significantly longer LOS and significantly higher rate of ischemic and hemorrhagic complications in SCG than in EVG (15). Similarly, in our study, the LOS was significantly shorter in EVG-b than in SCG-b. Shorter LOS increases patient comfort and prevents potential hospital-acquired infections. In addition, shorter LOS is associated with reduced treatment costs. Silva et al. compared surgical clipping and endovascular embolization in non-bleeding aneurysms in 21,595 patients and showed that the LOS in patients younger than 65 years was 6.3 days in SCG and 2.8 days in EVG, whereas that in patients older than 65 years was 8 days in SCG and 3 days in EVG (21). It has been reported that LOS in hospital is significantly lower after endovascular treatment (26). In our study, the average LOS was 2.8 days in EVG-nb. LOS has been correlated with previous studies.

A study that investigated residual aneurysms in association with non-bleeding aneurysms reported that both treatment methods were safe; however, the rate of residual aneurysms in EVG was higher than that in SCG (4). In the present study, aneurysms were closed in all patients treated surgically, and no residual aneurysms were detected. In EVG, residual aneurysms were detected in 6 of 92 aneurysms at the end of the first year, which indicates that the rate of residual aneurysms was $6.5 \%$. Residual aneurysms were most commonly found in middle cerebral artery (MCA) bifurcation aneurysms ranging from 7-10 mm. Mortimer et al. reported a mortality rate of $13.6 \%$, residual aneurysm rate of $8.1 \%$, and total occlusion rate of $91.4 \%$ in 175 bleeding and 44 non-bleeding MCA aneurysms treated endovascularly (20). In the present study, $17.8 \%$ of MCA aneurysms were detected at the bifurcation level, and $12.6 \%$ were detected in the M1 segment. In EVG, 40 aneurysms in 38 patients were treated. The rate of closure of MCA aneurysms was $90 \%$, and the rate of closure of all aneurysms was $93.4 \%$, similar to previous study findings.

In a study conducted by Koyanagi et al., 188 aneurysm cases were followed for an average of 10 years after endovascular treatment. Follow-up showed the presence of $\mathrm{SAH}$ in two patients. A giant aneurysm was detected in two patients who developed SAH. The authors stated that after the bleeding associated with intracranial aneurysms was treated endovascularly, patients generally showed a stable course (13). In the present study, SAH developed in two patients after the procedure. One patient who developed SAH had a giant internal carotid artery aneurysm, which measured $30 \mathrm{~mm}$. Both treated patients were discharged after 14 days, without neurological deficits. In giant aneurysms, SAH may occur after endovascular treatment. These findings revealed that caution must be exercised with the endovascular treatment approach in giant aneurysms.

In another study (2) that compared two treatment methods in ruptured aneurysms, in contrast with our results, the surgical group showed an advantage in terms of mortality, re-bleeding, and re-treatment. s. However, endovascular treatment was reported to be a better technique in terms of postoperative complications and rehabilitation. No significant difference was found in terms of postoperative death, bleeding, and modified Rankin score (mRS) >2 in unruptured aneurysms (10). Recent evidence suggests that surgical clipping leads to lower recurrence rates and is associated with a higher incidence of complete occlusion, whereas endovascular therapy is associated with shorter LOS and a lower complication rate (12).

Our results suggest that endovascular therapy reduces LOS and increases patient comfort. Similar findings have been reported in previous studies. A study that compared healthrelated quality of life between the two treatment modalities over a 10-year period (9) reported that health-related quality of life after treatment of a ruptured intracranial aneurysm was better after endovascular treatment than after surgical clipping.

In the last two decades, the incidence of endovascular treatment in ruptured and unruptured aneurysms has significantly increased, whereas the incidence of surgical clipping in ruptured aneurysms has decreased $(8,14)$. Beydoun et al. reported that the perioperative ischemic stroke rate in unruptured aneurysms was lower in those who underwent stent- and balloon-assisted coil therapy than in those who underwent surgical clipping alone (5). In a study that compared endovascular treatment with surgical clipping and low-profile visualized intraluminal support stent-assisted coiling in blood blister-like aneurysms, it was reported that the endovascular group had a better prognosis in terms of hospital stay and $\mathrm{mRS}$ at discharge. Moreover, the risk of complications due to a second operation and procedure was found to be lower than that in surgical clipping (25). In small ruptured aneurysms, both treatment methods were found to be equivalent in terms of procedural complications and neurological outcomes, similar to our findings (6). Complementary application of both endovascular treatment and open surgery to the basilar artery facilitates individualized treatment planning of basilar artery aneurysms. By exploiting the strengths of both techniques, equivalent clinical results and technical proficiency can be achieved with both methods (24). 


\section{Limitations}

The design of this study was retrospective, and evaluations were performed according to the results after the first year. This study was also a single-center study and represents only the experiences at our clinic.

\section{CONCLUSION}

Cerebral aneurysms are a critical health problem. Owing to technological developments, the rate of aneurysm detection has increased. Aneurysms must be treated to prevent SAH. Both endovascular treatment and surgical clipping are effective and safe methods, and both have advantages and disadvantages. Therefore, an appropriate choice should be made according to patient needs.

\section{- REFERENCES}

1. Abecassis IJ, Zeeshan Q, Ghodke BV, Levitt MR, Ellenbogen $R G$, Sekhar LN: Surgical versus endovascular management of ruptured and unruptured intracranial aneurysms: Emergent issues and future directions. World Neurosurg 136:17-27, 2020

2. Ahmed SI, Javed G, Bareeqa SB, Samar SS, Shah A, Giani A, Aziz Z, Tasleem A, Humayun SH: Endovascular coiling versus neurosurgical clipping for aneurysmal subarachnoid hemorrhage: A systematic review and meta-analysis. Cureus 11:e4320, 2019

3. Ajiboye N, Chalouhi N, Starke RM, Zanaty M, Bell R: Unruptured cerebral aneurysms: Evaluation and management. ScientificWorldJournal 2015:954954, 2015

4. Bernat AL, Clarencon F, Andre A, Nouet A, Clemenceau S, Sourour NA, Di Maria F, Degos V, Golmard JL, Cornu P, Boch $A L$ : Risk factors for angiographic recurrence after treatment of unruptured intracranial aneurysms: Outcomes from a series of 178 unruptured aneurysms treated by regular coiling or surgery. J Neuroradiol 44:298-307, 2017

5. Beydoun HA, Beydoun M, Zonderman A, Eid SM: Perioperative ischemic stroke in unruptured intracranial aneurysm surgical or endovascular therapy. Cureus 12:e7645, 2020

6. Catapano JS, Nguyen CL, Frisoli FA, Sagar S, Baranoski JF, Cole TS, Labib MA, Whiting AC, Ducruet AF, Albuquerque FC, Lawton MT: Small intracranial aneurysms in the Barrow Ruptured Aneurysm Trial (BRAT). Acta Neurochir (Wien) 163:123-129, 2021

7. Fotakopoulos G, Tsianaka E, Fountas K, Makris D, Spyrou M, Hernesniemi J: Clipping versus coiling in anterior circulation ruptured intracranial aneurysms: A meta-analysis. World Neurosurg 104:482-488, 2017

8. Golnari P, Nazari P, Garcia RM, Weiss H, Shaibani A, Hurley MC, Ansari SA, Potts MB, Jahromi BS: Volumes, outcomes, and complications after surgical versus endovascular treatment of aneurysms in the United States (1993-2015): Continued evolution versus steady-state after more than 2 decades of practice. J Neurosurg 134:848-861, 2021
9. Hua X, Gray A, Wolstenholme J, Clarke P, Molyneux AJ, Kerr RSC, Clarke A, Sneade M, Rivero-Arias O: Survival, dependency, and health-related quality of life in patients with ruptured intracranial aneurysm: 10-year follow-up of the united kingdom cohort of the international subarachnoid aneurysm trial. Neurosurgery 88:252-260, 2021

10. Huang C, You C: Clipping versus coiling in the management of unruptured aneurysms with multiple risk factors. World Neurosurg 126:e545-e549, 2019

11. Jiang Z, Chen Y, Zeng C, Feng J, Wan Y, Zhang X: Neurosurgical clipping versus endovascular coiling for patients with intracranial aneurysms: A systematic review and meta-analysis. World Neurosurg 138:e191-e222, 2020

12. Kang XK, Guo SF, Lei Y, Wei W, Liu HX, Huang LL, Jiang QL: Endovascular coiling versus surgical clipping for the treatment of unruptured cerebral aneurysms: Direct comparison of procedure-related complications. Medicine (Baltimore) 99:e19654, 2020

13. Koyanagi $M$, Ishii $A$, Imamura $H$, Satow $T$, Yoshida $K$, Hasegawa H, Kikuchi T, Takenobu Y, Ando M, Takahashi JC, Nakahara I, Sakai N, Miyamoto S: Long-term outcomes of coil embolization of unruptured intracranial aneurysms. J Neurosurg 129(6):1492-1498, 2018

14. Luther E, McCarthy DJ, Brunet MC, Sur S, Chen SH, Sheinberg D, Hasan D, Jabbour P, Yavagal DR, Peterson EC, Starke RM: Treatment and diagnosis of cerebral aneurysms in the postInternational Subarachnoid Aneurysm Trial (ISAT) era: Trends and outcomes. J Neurointerv Surg 12:682-687, 2020

15. McDonald JS, McDonald RJ, Fan J, Kallmes DF, Lanzino G, Cloft HJ: Comparative effectiveness of unruptured cerebral aneurysm therapies: Propensity score analysis of clipping versus coiling. Stroke 44:988-994, 2013

16. McDougall CG, Spetzler RF, Zabramski JM, Partovi S, Hills NK, Nakaji P, Albuquerque FC: The barrow ruptured aneurysm trial. J Neurosurg 116:135-144, 2012

17. Metayer T, Terrier L, de Schlichting E, Lechanoine F, Bougaci N, Derrey S, Barbier C, Papagiannaki C, Ashraf A, Tahon F, Leplus A, Naveau M, Gay E, Emery E, Lonjon M, Vivien D, Gaberel T: Rate of rebleed and retreatment of previously treated intracranial aneurysms. World Neurosurg 148:e650-e657, 2021

18. Molyneux A, Kerr R, Stratton I, Sandercock P, Clarke M, Shrimpton J, Holman R: International Subarachnoid Aneurysm Trial (ISAT) of neurosurgical clipping versus endovascular coiling in 2143 patients with ruptured intracranial aneurysms: A randomized trial. J Stroke Cerebrovasc Dis 11:304-314, 2002

19. Molyneux AJ, Birks J, Clarke A, Sneade M, Kerr RS: The durability of endovascular coiling versus neurosurgical clipping of ruptured cerebral aneurysms: 18 year follow-up of the UK cohort of the International Subarachnoid Aneurysm Trial (ISAT). Lancet 385:691-697, 2015

20. Mortimer AM, Bradley MD, Mews P, Molyneux AJ, Renowden SA: Endovascular treatment of 300 consecutive middle cerebral artery aneurysms: Clinical and radiologic outcomes. AJNR Am J Neuroradiol 35:706-714, 2014 
21. Silva NA, Shao B, Sylvester MJ, Eloy JA, Gandhi CD: Unruptured aneurysms in the elderly: Perioperative outcomes and cost analysis of endovascular coiling and surgical clipping. Neurosurg Focus 44:E4, 2018

22. Spetzler RF, McDougall CG, Zabramski JM, Albuquerque FC, Hills NK, Russin JJ, Partovi S, Nakaji P, Wallace RC: The barrow ruptured aneurysm trial: 6-year results. J Neurosurg 123:609-617, 2015

23. Vlak MH, Algra A, Brandenburg R, Rinkel GJ: Prevalence of unruptured intracranial aneurysms, with emphasis on sex, age, comorbidity, country, and time period: A systematic review and meta-analysis. Lancet Neurol 10:626-636, 2011
24. Winkler EA, Lee A, Yue JK, Raygor KP, Rutledge WC, Rubio RR, Josephson SA, Berger MS, Raper DMS, Abla AA: Endovascular embolization versus surgical clipping in a single surgeon series of basilar artery aneurysms: A complementary approach in the endovascular era. Acta Neurochir (Wien) 163(5):1527-1540, 2021

25. Wu YQ, Li LZ, Wang ZY, Zhang T, Xu M, Cheng MX: Endovascular Intervention with a low-profile visualized intraluminal support stent versus surgical clipping for blood blister-like aneurysms: A retrospective study. Clin Neuroradiol 31(2):417-424, 2021

26. Zhang X, Tang H, Huang Q, Hong B, Xu Y, Liu J: total hospital costs and length of stay of endovascular coiling versus neurosurgical clipping for unruptured intracranial aneurysms: Systematic review and meta-analysis. World Neurosurg 115:393-399, 2018 